\title{
Rotational-Slice-Based Prostate Segmentation Using Level Set with Shape Constraint for 3D End-Firing TRUS Guided Biopsy
}

\author{
Wu Qiu, Jing Yuan, Eranga Ukwatta, David Tessier, and Aaron Fenster \\ Imaging Research Laboratories, Robarts Research Institute, 100 Perth, London, Ontario, \\ Canada, N6A 5k8 \\ \{WQiu, JYuan, EUkwatta, DTessier,AFenster\} @imaging.robarts.ca
}

\begin{abstract}
Prostate segmentation in 3D ultrasound images is an important step in the planning and treatment of 3D end-firing transrectal ultrasound (TRUS) guided prostate biopsy. A semi-automatic prostate segmentation method is presented in this paper, which integrates a modified distance regularization level set formulation with shape constraint to a rotational-slice-based 3D prostate segmentation method. Its performance, using different metrics, has been evaluated on a set of twenty $3 \mathrm{D}$ patient prostate images by comparison with expert delineations. The volume overlap ratio of $93.39 \pm 1.26 \%$ and the mean absolute surface distance of $1.16 \pm 0.34 \mathrm{~mm}$ were found in the quantitative validation result.
\end{abstract}

Keywords: prostate segmentation, 3D ultrasound, level set, shape constraint, rotational volume reslicing.

\section{Introduction}

Prostate cancer is the most frequent cancer in men in the United States and Europe with an incidence that reaches more than $25 \%$ of the new cases of cancers[14]. Transrectal ultrasound (TRUS) is currently the most commonly used imaging modality for image-guided biopsy and therapy of prostate cancer due to its real-time nature, low cost, and simplicity[4]. Moreover, accurate segmentation of the whole prostate from the TRUS image plays a key role in biopsy and therapy planning[3[18]. In addition, it allows for surface-based registration between the TRUS and other imaging modalities (e.g., MRI) during the image-guided intervention[6]. Although manual segmentation of the prostate boundary in 3D US images is possible, it is arduous and time consuming. However, automated and semi-automated prostate segmentation has challenges due to the presence of speckle noise, calcifications, nearby organs, missing edges or similarities between inner and outer texture of the prostate. In addition, prostate segmentation from 3D end-firing TRUS images is more challenging than from 3D side-firing TRUS images due to a few main reasons: (a) end-firing images are more inhomogeneous; (b) the prostate is located arbitrarily in the $3 \mathrm{D}$ volume, not just approximately in the center of the volume, thus, the localization uncertainty increases the difficulty of automatic and semi-automatic segmentation methods; and (c) the prostate is deformed differently in different images due to varying pressure of the end-firing transducer during the biopsy procedure. 
3D prostate segmentation methods can be categorized into two classes: direct 3D segmentation and propagation of the $2 \mathrm{D}$ slice-based segmentation. In terms of the first class, the user initializs a 3D deformable surface in multiple 2D slices of the prostate, then the initial 3D mesh is automatically refined by forces characterized by the image gradient and smoothness of the contour. Hu et al.[7] manually defined an ellipsoid that served as an initial guess. The estimated outline is then automatically deformed to better fit the prostate boundary. Abolmaesumi et al.[1] proposed to assimilate the prostate contour to the trajectory of a moving object that they tracked with an interacting multiple model probabilistic data association filter (IMM/PDAF). Following an initialization that consisted of selecting the base and apex axial slices, and seven landmarks. Mahadavi et al.[12] fitted a warped, tapered ellipsoid to the prostate using the edge points detected with the IMM/PDA filter. Carole et al.[5] presented a semi-automatic method based on discrete dynamic contour and optimal surface detection to segment the prostate in high intensity focused ultrasound(HIFU) therapy. This kind of method works well for high contrast 3D US images, but is time consuming and requires many user interactions that can lead to observer variability. Other methods are based on the slice-based propagation approach. Within slice-based segmentation, the result is iteratively propagated onto adjacent slices and then refined. Yan et al.[17] proposed a method combining local shape statistics and discrete deformable modal (DDM) to segment 2D video sequences of prostates. These approaches can give favorable results, however, they encounter difficulties in handling slices that are nearly tangent to the prostate surface (3D boundary), such as in the base and apex areas of the prostate. To overcome these issues, some researchers unfolded the 3D image in a rotational manner[11]. Wang et al.[16] resliced the $3 \mathrm{D}$ volume rotationally. All 2D slices pass through a common axis, approximately centered in the prostate, around which they are evenly radially spaced. DDM was then applied on each resliced image. Ding et al.[3] corrected them automatically by imposing a continuity constraint on the size of successive contours using an auto-regressive model. There does, however, remain the possibility that the contours may suffer from the accumulated error and "leak" at locations of weak edges[3].

To address the challenges involved with prostate segmentation, a level set based method is proposed in this paper, which has been used in the $3 \mathrm{D}$ side-firing TRUS guided prostate biopsy system. The key contribution of this study is that it incorporated the level set method with constraint shape information and high-level user interaction into a rotational-slice-based segmentation approach. It can potentially decrease the accumulated segmentation errors that occur in the propagation based method while preserving the accuracy .

\section{Method}

\subsection{Overview of Approach}

The proposed rotational-slice-based prostate 3D segmentation method is divided into three steps: first, selecting two points manually (red points in Fig[1(a)) on the long axis of the boundary on the coronal view of prostate. The initial slice and rotational axis is determined by these two points. The plane (blue slice in Fig. 1 a)) that includes these two points and is perpendicular to the coronal view is the approximate transverse view 


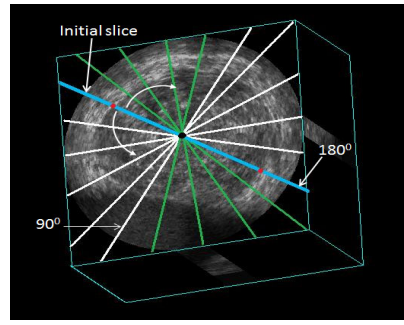

(a)

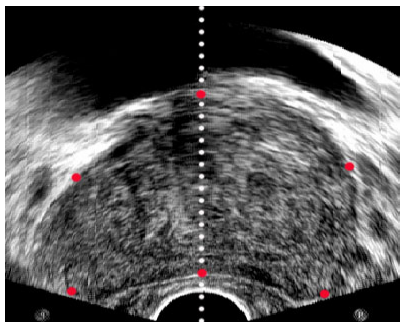

(b)

Fig. 1. Overview of approach. (a)reslicing the volume, (b)initializing boundary points.

of the prostate, which is assumed as the initial slice of the propagation method . The line on the transverse view perpendicularly bisecting the segment determined by those two selected points is identified as the rotational axis. Second, rotational reslicing of the 3D image of the prostate into $N$ slices about the identified rotational axis is performed. All slices intersect along the axis approximately and have an equal angular spacing. The step angle was six degrees in our experiments resulting in a $3 \mathrm{D}$ volume that was resliced into 30 slices $(N=30)$. Third, four to eight initialization points (red points in Fig. 1(b))are manually chosen from the boundary of the prostate in the initial slice in which the initial contour is estimated using cardinal spline interpolation. A level set method is then used to deform this initial contour, which will be described in detail in section 2.2. Finally, the deformed contour in the initial slice is then propagated to its adjacent slice in a clockwise(green slices in Fig. 1a)) and counter-clockwise (white slices in Fig. 1(a))direction, which is used as the initial contour before refining. This procedure is repeated until the contours in all 2D slices of the 3D image are segmented. A 3D prostate surface mesh is reconstructed from the contours in all 2D slices.

The detailed propagation will now be explained. The first segmented contour for the initial slice is used as the shape constraint for slice 1 to $M$ and slice $N$ to slice $N-M$ independently ( $M=3$ in our experiments). The resulting shapes $\left\{S_{i} \mid i=1, . ., M\right\}$ for the clockwise direction and $\left\{\hat{S}_{i} \mid i=N, . ., N-M\right\}$ for the counter clockwise direction

will be stored; the mean shape $\bar{S}_{M}$ and $\overline{\hat{S}}_{M}$ will be calculated, which is used as the shape constraint of the level set formulation for segmenting the next slice. From the slice $M+1$, the obtained prostate contour will be added into set $S_{i}$, shape $S_{1}$ will be removed from set $S_{i}$, and the new mean shape $\bar{S}_{M}$ of $S_{i}$ will be recalculated. The updated $\bar{S}_{M}$ will be given as the constraint shape for the next slice. This process is repeated until the segmentation is finished in the clockwise direction. The same procedure is conducted for the segmentation in the counter clockwise direction.

\subsection{Segmentation Using Level Set}

A distance regularization level set formulation (DRLSE)[10] method is modified to segment the prostate contour in each 2D slice. Let $\phi: \Omega \rightarrow \Re$ be a level set formulation (LSF) defined on a domain $\Omega$. An energy functional $\varepsilon(\phi)$ is defined as:

$$
\varepsilon(\phi)=\mu R_{p}(\phi)+\varepsilon_{e x t}(\phi)
$$


where $\mu>0$ is a constant and $R_{p}(\phi)$ is the level set regularization term, defined by

$$
R_{p}(\phi) \triangleq \int_{\Omega} p(|\nabla \phi|) d x
$$

where $p$ is a potential (or energy density) function $p:[0, \infty) \rightarrow \Re$. External energy term $\varepsilon_{\text {ext }}(\phi)$ is defined in the following. The minimization of the energy $\varepsilon(\phi)$ can be achieved by solving a level set evolution equation.

For an LSF $\phi: \Omega \rightarrow \Re$, an external energy function $\varepsilon_{\text {ext }}(\phi)$ is defined by

$$
\varepsilon_{\text {ext }}(\phi)=\lambda L_{g}(\phi)+\alpha A_{g}(\phi)+\beta S(\phi)+\gamma T(\phi)+\nu A(\phi)
$$

where $\lambda>0$ and $\alpha \in \Re$ are the coefficients of the energy functionals $L_{g}(\phi)$ and $A_{g}(\phi)$, which are given by

$$
L_{g}(\phi) \triangleq \int_{\Omega} g \delta(\phi)|\nabla \phi| d x
$$

and

$$
A_{g}(\phi) \triangleq \int_{\Omega} g H(-\phi) d x
$$

where $g$ is an edge indicator function defined by $g \triangleq \frac{1}{1+\left|\nabla G_{\sigma} * I\right|^{2}}$. The Dirac delta function $\delta$ and Heaviside function in $H \mathrm{Eq} 4$ and $\mathrm{Eq} 5$ are approximated by the following smooth function $\delta_{\varepsilon}$ and $H_{\varepsilon}$, respectively, as in many level set methods[2]13]. The energy $L_{g}(\phi)$ computes the line integral of the function $g$ along the zero level contour of $\phi$. The energy $A_{g}(\phi)$ calculates the weighted area of the region $\Omega_{\phi}^{-} \triangleq x: \phi(x)<0$, which is introduced to speed up the motion of the zero level contour in the evolution process. $S(\phi), A(\phi)$ and $T(\phi)$ are shape constraint energy, anchor point energy and local region-based energy, respectively.

Since the contour to be segmented is not far from the contour propagated from the previous slice, a shape constraint is imposed to discourage the evolved contour to leak in the region with a weak edge or without an edge. This shape constraint energy $S(\phi)$ [15]is defined by

$$
S(\phi)=\int_{\Omega} \delta(\phi(x)) B_{S}(x) d x
$$

where

$$
B_{S}(x)=\left\{\begin{array}{rrr}
0 & \text { if } & \min _{\hat{x}} D(x, \hat{x})<d_{S} \\
D(x, \hat{x}) & \text { otherwise }
\end{array}\right.
$$

$D(x, \hat{x})=\|x-\hat{x}\| . \hat{x} \in \Omega$ is an independent spatial variable, which is given by the constraint shape (described in section 2.1). $d_{S}$ is the separation distance from the current evolving LSF to the constraint shape. This energy is nonzero when the evolving LSF moves farther from the constraint shape of any distance greater than $d_{S}$.

Let $\hat{y} \in \Omega$ be another independent spatial variable, the local region-based energy $T(\phi)[9]$ is

$$
\begin{aligned}
T(\phi(x))=\int_{\Omega} \delta(\phi(x)) & \int_{\Omega} B_{L}(x, \hat{y})\left[H(\phi(\hat{y}))(I(\hat{y}))-u_{x}\right)^{2} \\
& \left.\left.+(1-H(\phi(\hat{y})))(I(\hat{y}))-v_{x}\right)^{2}\right] d \hat{y} d x
\end{aligned}
$$


where

$$
B_{L}(x, \hat{y})=\left\{\begin{array}{lrr}
1 & \text { if } & \|x-\hat{y}\|<r_{L} \\
0 & \text { otherwise }
\end{array}\right.
$$

is used to define a circular-shaped local region with localizing radius $r_{L}$.

$u_{x}=\int_{\Omega} B_{L}(x, \hat{y}) H(\phi(\hat{y})) I(\hat{y}) d \hat{y} / \int_{\Omega} B_{L}(x, \hat{y}) H(\phi(\hat{y})) d \hat{y}$ and $v_{x}=\int_{\Omega} B_{L}(x, \hat{y})(1-$ $H(\phi(\hat{y}))) I(\hat{y}) d \hat{y} / \int_{\Omega} B_{L}(x, \hat{y})(1-H(\phi(\hat{y}))) d \hat{y}$ are the mean image intensities of the interior and exterior of the active contour, respectively, within the region defined by $B_{L}(x, \hat{y}) \cdot r_{L}$ determines the degree of blending local statistics around the boundary to global statistics of the image.

The energy $A(\phi)$ given by Eq. 10][15] encourages the evolving contour to pass through each anchor point $x_{A}^{i}$ if the evolving contour is within a distance $r_{A}$ to the anchor point. Anchor points in the first slice are the initial boundary points placed by users. During the propagation, two anchor points are selected, which act as the intersection points of the rotational axis and the segmented contour in the first slice. When the contour points are away from the anchor point by more than $r_{A}$, there is no influence by this energy.

$$
A(\phi)=\sum_{i=1}^{N_{p}} \int_{\Omega} \delta(\phi(x)) B_{A}^{i}(x)\left(\phi(x)-\phi\left(x_{A}^{i}\right)\right)^{2} d x
$$

where

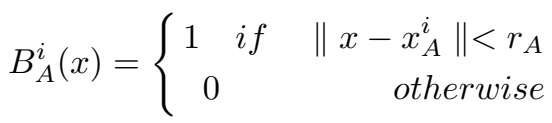

is used to define a circular-shaped region around the anchor point with radius $r_{A} ; N_{p}$ is the number of anchor points.

By taking the first variation of the Eq1 with respect to $\phi$, the following evolving equation will be obtained:

$$
\begin{array}{r}
\frac{\partial \phi(x)}{\partial t}=\mu \operatorname{div}\left(d_{p}(|\nabla \phi|) \nabla \phi\right)+\delta(\phi(x))\left\{\lambda \operatorname{div}\left(g \frac{\nabla \phi}{|\nabla \phi|}\right)+\alpha g+\beta B_{S}(x)\right. \\
\left.+\gamma \int_{\Omega} B_{L}(x, \hat{y}) \delta\left(\phi(\hat{y})\left[(I(\hat{y}))-u_{x}\right)^{2}-(I(\hat{y}))-v_{x}\right)^{2}\right] d \hat{y} \\
\left.+\nu \sum_{i=1}^{N_{p}} B_{A}^{i}(x)\left(\phi(x)-\phi\left(x_{A}^{i}\right)\right)\right\}
\end{array}
$$

\section{Experimental Results}

All testing images were acquired with a rotational scanning 3D TRUS imaging system using a commercially available end-firing TRUS transducer (Philips, Bothell WA)[8], which is developed for 3D US guided prostate biopsy procedure. The size of each 3D image is $448 \times 448 \times 350$ voxels of size $0.19 \times 0.19 \times 0.19 \mathrm{~mm}^{3}$. Twenty five patient images were tested in this paper, which are divided into two sets. Five were used to optimize the parameter values of the algorithm, and the other twenty images were used 
Table 1. Parameters and their optimized values

\begin{tabular}{lccccccccc}
\hline & $\lambda$ & $\mu$ & $\alpha$ & $\beta$ & $\gamma$ & $\nu$ & $d_{S}$ (pixels) & $r_{L}$ (pixels) & $r_{A}$ (pixels) \\
\hline values & 8 & 0.04 & -5 & 10 & 5 & 5.5 & 8 & 20 & 5 \\
\hline
\end{tabular}

to validate the proposed method. The parameter values were empirically chosen. Afterwards, the parameters were optimized sequentially by changing a single parameter at a time while holding other parameters fixed. Table 1 shows the optimized values of the parameters used in our experiments, which were kept constant during the validation experiments. The performance of the proposed method has been evaluated qualitatively by visual comparison with the expert-delineated contours. One reconstructed prostate surface (green surface after segmentation) is demonstrated in Fig. 2, which is superimposed on the manual surface (red one). Volume-based metrics: sensitivity(Se),

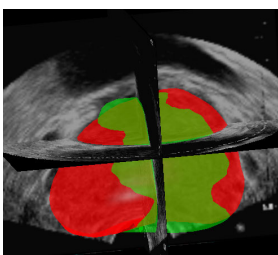

(a)

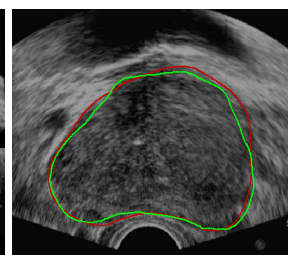

(b)

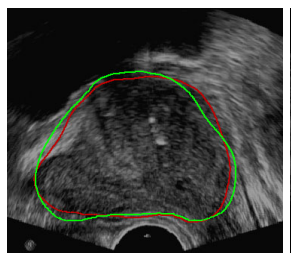

(c)

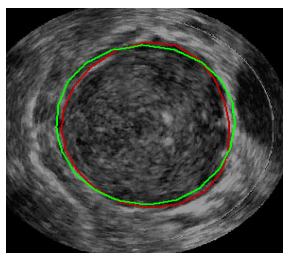

(d)

Fig. 2. Prostate segmentation result (green line) compared to manual segmentation(red line). (a)superimposed surface, (b)transverse view, (c)sagittal view, and (c) coronal view .

dice similarity coefficient (DSC) and volume difference $\left(V_{\text {diff } f}\right)$, and distance-based metrics: the mean absolute surface distance(MAD) and maximum absolute surface distance(MAXD) [5]were used to validate our segmentation method $\left(M_{L S}\right)$ quantitatively compared to manual segmentation and other rotational-slice-based methods (Wang's $\operatorname{method}\left(M_{C W}\right)[16]$ and Ding's method $\left.\left(M_{C C W}\right)[3]\right)$. The coefficient of variation $(C V)$ [19] of volume overlap rate was used to evaluate the intraobserver variability of our method.

The validation results in Table 2 show that our method can obtain a sensitivity of $94.28 \pm 2.18 \%$ and a DSC of $93.39 \pm 1.26 \%$ better than $M_{C W}(85.24 \pm 6.28 \%, 84.29 \pm$ $4.18 \%)$ and $M_{C C W}(88.33 \pm 4.58 \%, 87.18 \pm 3.37 \%) . M A D$ of $1.16 \pm 0.34 \mathrm{~mm}$ and $M A X D$ of $3.06 \pm 0.76 \mathrm{~mm}$ of our method were obtained comparable to $1.95 \pm 0.74 \mathrm{~mm}$ and $4.67 \pm 1.84 \mathrm{~mm}$ of $M_{C W}, 1.59 \pm 0.67 \mathrm{~mm}$ and $4.2 \pm 1.56 \mathrm{~mm}$ of $M_{C C W}$. The comparison with manual segmentation on volume difference $\left(V_{\text {diff }}\right)$ demonstrates a volume error of $2.45 \pm 1.75 \mathrm{~cm}^{3}$ on the entire prostate. The intraobserver variability experiments shows that our proposed method gave a $C V$ of $2.5 \%$. To validate the variability introduced by the manual initialization (initial slice and initial points), an inter-observer variability test was performed. Ten images were also segmented by three untrained observes who were blinded to patient identity. The proposed method yielded a DSC of $93.1 \pm 1.6 \%, 93.6 \pm 1.5 \%$ and $92.4 \pm 1.7 \%$, and $C O V$ of $1.7 \%, 1.6 \%$ and $1.8 \%$, 
respectively. The proposed method was developed in Matlab. The segmentation time of our method was found to be about $45 \mathrm{~s}$ on a desktop computer with a core 2 CPU (2.66 $\mathrm{GHz}$ ) in addition to 30 s for initialization. A total segmentation time was found to be less than 1.5 minute.

Ten images randomly selected were used to test the sensitivity of our method to some parameters. The effect of $d_{s}, r_{L}$, and $r_{A}$ on the segmentation accuracy $(D S C)$ were only observed since they are dependent on the values of $\beta, \gamma$ and $\nu$ that were kept constant (More details on how to choose these three parameters refer to [10]). As a result, DSC goes up slightly when $d_{s}$ increases from 0 to 4 pixels, and it goes down when $d_{s}$ is greater than 3 pixels. The range from 3 to 5 pixels for $d_{s}$ is suggested. Our method presents the growth of DSC when the radius $r_{L}$ of the local region increases from 0 to 10. It demonstrates less standard deviation of DSC at the expense of a decreasing DSC when $r_{L}$ is greater than 20. In addition, DSC displays a downward trend with the growing radius of the anchor point. $r_{A}$ varying from 0 to 5 pixels is capable of giving a good segmentation accuracy.

Table 2. Overall performance results

\begin{tabular}{|c|c|c|c|c|c|}
\hline & $\mathrm{Se}(\%)$ & $\operatorname{DSC}(\%)$ & $\operatorname{MAD}(m m(v x))$ & $\operatorname{MAXD}(\operatorname{mm}(\mathrm{vx}))$ & $V_{\text {diff }}\left(\mathrm{cm}^{3}\right)$ \\
\hline$M_{L S}$ & $94.28 \pm 2.18$ & $93.39 \pm 1.26$ & $\begin{array}{l}1.16 \pm 0.34 \\
(6.13 \pm 1.8)\end{array}$ & $\begin{array}{c}3.06 \pm 0.76 \\
(16.1 \pm 4.02)\end{array}$ & $2.45 \pm 1.75$ \\
\hline$M_{C W}$ & \pm 6.28 & $84.29 \pm 4.18$ & $\begin{array}{c}1.95 \pm 0.74 \\
(10.25 \pm 3.89)\end{array}$ & $\begin{array}{c}4.67 \pm 1.84 \\
(24.56 \pm 9.7)\end{array}$ & 1.03 \\
\hline$M_{C C W}$ & $88.33 \pm 4.58$ & $87.18 \pm 3.37$ & $\begin{array}{c}1.59 \pm 0.67 \\
(8.38 \pm 3.54)\end{array}$ & $\begin{array}{c}4.2 \pm 1.56 \\
(22.1 \pm 8.2)\end{array}$ & $4.22 \pm 3.95$ \\
\hline
\end{tabular}

\section{Conclusion}

A rotational-slice-based segmentation method, with a modified level set, has been proposed in this paper for a semi-automatic segmentation of the prostate in 3D end-firing TRUS images. It has been evaluated on a patient dataset obtained during a 3D end-firing TRUS guided prostate biopsy procedure. The resulting segmentations have been compared with manual expert delineations using different metrics (Se, DSC, MAD, MAXD, and volume difference). It is found that the proposed method is accurate, robust and computationally efficient.

\section{References}

1. Abolmaesumi, P., Sirouspour, M.R.: An interacting multiple model probabilistic data association filter for cavity boundary extraction from ultrasound images. IEEE Trans. Med. Imag. 23(6), 772-784 (2004)

2. Chan, T.F., Vese, L.A.: Active contours without edges. IEEE Trans Img. Process. 10(2), 266277 (2001)

3. Ding, M., Chiu, B., Gyacskov, I., Yuan, X., Drangova, M., Downey, D.B., Fenster, A.: Fast prostate segmentation in $3 \mathrm{~d}$ trus images based on continuity constraint using an autoregressive model. Med. Phys. 34(11), 4109-4125 (2007) 
4. Fenster, A., Downey, D.B.: Three-dimensional ultrasound imaging and its use in quantifying organ and pathology volumes. Anal. Bioanal. Chem. 377(6), 982-989 (2003)

5. Garnier, C., Bellanger, J.J., Wu, K., Shu, H., Costet, N., Mathieu, R., de Crevoisier, R., Coatrieux, J.L.: Prostate segmentation in hifu therapy. IEEE Trans. Med. Imag. 30(3), 792-803 (2011)

6. Ghose, S., Oliver, A., Martí, R., Lladó, X., Freixenet, J., Vilanova, J.C., Meriaudeau, F.: Texture Guided Active Appearance Model Propagation for Prostate Segmentation. In: Madabhushi, A., Dowling, J., Yan, P., Fenster, A., Abolmaesumi, P., Hata, N. (eds.) MICCAI 2010. LNCS, vol. 6367, pp. 111-120. Springer, Heidelberg (2010)

7. Hu, N., Downey, D.B., Fenster, A., Ladak, H.M.: Prostate boundary segmentation from 3d ultrasound images. Med. Phys. 30(7), 1648-1659 (2003)

8. Bax, J., Cool, D., Gardi, L., Knight, K., Smith, D., Montreuil, J., Sherebrin, S., Romagnoli, C., Fenster, A.: Mechanically assisted 3d ultrasound guided prostate biopsy system. Med. Phys. 35(12), 5397, 5401 (2008)

9. Lankton, S., Tannenbaum, A.: Localizing region-based active contours. IEEE Trans. Imag. Process. 17(11), 2029-2039 (2008)

10. Li, C., Xu, C., Gui, C., Fox, M.D.: Distance regularized level set evolution and its application to image segmentation. IEEE Trans. Imag. Process. 19(12), 3243-3254 (2010)

11. Li, K., Wu, X., Chen, D.Z., Sonka, M.: Optimal surface segmentation in volumetric images-a graph-theoretic approach. IEEE Trans. Pattern Anal. Mach. Intell. 28(1), 119-134 (2006)

12. Mahdavi, S.S., Moradi, M., Wen, X., Morris, W.J., Salcudean, S.E.: Evaluation of visualization of the prostate gland in vibro-elastography images. Medical Image Analysis 15(4), 589-600 (2011)

13. Malladi, R., Sethian, J.A., Vemuri, B.C.: Shape modeling with front propagation: A level set approach. IEEE Trans. Pattern Anal. Mach. Intell. 17(2), 158-175 (1995)

14. Soc, A.C.: Cancer facts and figures (2010), http: / / www . cancer.org

15. Ukwatta, E., Awad, J., Ward, A.D., Buchanan, D., Samarabandu, J., Parraga, G., Fenster, A.: Three-dimensional ultrasound of carotid atherosclerosis: semiautomated segmentation using a level set-based method. Med. Phys. 38(5), 2479-2493 (2011)

16. Wang, Y., Cardinal, H.N., Downey, D.B., Fenster, A.: Semiautomatic three-dimensional segmentation of the prostate using two-dimensional ultrasound images. Med. Phys. 30(5), 887897 (2003)

17. Yan, P., Xu, S., Turkbey, B., Kruecker, J.: Adaptively learning local shape statistics for prostate segmentation in ultrasound. IEEE Trans. Bio. Med. Eng. 58(3), 633-641 (2011)

18. Zhan, Y., Shen, D.: Deformable segmentation of 3-d ultrasound prostate images using statistical texture matching method. IEEE Trans. Med. Imag. 25(3), 256-272 (2006)

19. Zou, K.H., Mcdermott, M.P.: Higher-moment approaches to approximate interval estimation for a certain intraclass correlation coefficient. Statistics in Medicine 18(15), 2051-2061 (1999) 